\title{
Effects of Gut Distension on Auerbach's Plexus and Intestinal Muscle
}

\author{
Syomatu YoKoYAmA* and Tsuyoshi OzAKI \\ Department of Physiology, Fukushima Medical College, Fukushima, 960 Japan
}

\begin{abstract}
Using a peeled longitudinal muscle strip which was attached to an intestinal segment of rabbit, the effects of gut distension upon the activities of both the neurons within Auerbach's plexus and the intestinal muscle were investigated. Spontaneous neuronal and muscular discharges were recorded by means of a micropipette electrode which was placed on a node of the exposed Auerbach's plexus. Contractions of the peeled longitudinal muscle strip were simultaneously recorded. Gut distension caused an excitatory or inhibitory effect on mechanosensitive neurons. The neurons whose spontaneous discharges showed a frequency increase during muscle contraction and a frequency decrease during muscle relaxation at zero intraluminal pressure responded to gut distension as well as to stretching of the longitudinal muscle with excitation. The neurons which did not show any correlation in thier discharge frequency with muscle contractions responded to gut distension as well as to stretching of the longitudinal muscle with inhibition. Additional neurons were found in small numbers whose spontaneous activity did not relate in frequency to muscle contractions and did not change by gut distension. In all cases of gut distension the excitatory effect on longitudinal muscle as well as on intestinal segment, namely the peristaltic movement, were observed. Removal of mucosa did not affect the abovedescribed effects of gut distension. It was concluded that gut distension causes an excitatory effect on specific mechanosensitive neurons in Auerbach's plexus which regulate the contractions of intestinal muscle and produce the peristaltic movements.
\end{abstract}

BAYLISS and STARLING (1899) reported that the peristaltic movements of the small intestine are true, coordinated reflexes, initiated by mechanical stimulation of the intestine and carried out by the local nervous mechanism within Auerbach's plexus. Trendelenburg (1917) showed that the peristaltic movements of the excised small intestine of guinea pig or rabbit were caused by elevation of in-

Received for publication August 25, 1979

横山正松, 尾崎 毅

* Present address: Neurophysiology Laboratory, Department of Pharmacology, Loyola University, Stritch School of Medicine, Maywood, Ill. 60513, U.S.A. 
traluminal pressure. HuKuHARA et al. (1958), using the denervated small intestine of dog, proved that the local mechanical or chemical stimulation of the intestinal mucosa elicited peristaltic movements which were abolished by application of hexamethonium. Crema et al. (1970), and Frigo and LeCCHINI (1970) also proved that the localized distension of the colon of cat or guinea pig caused the propulsive movement of colon which was abolished by application of hexamethonium or atropine.

It can be thought from the above-mentioned studies that Auerbach's plexus acts as the reflex arc when the peristaltic movement is caused by gut distension or by mechanical or chemical stimulation of the intestinal mucosa. The properties of excitation conduction in the network of Auerbach's plexus were investigated by YoкоYama et al. (1977) and those of efferent pathways in the reflex arc of Auerbach's plexus were partly clarified by YoKoyAMA and OzAKI (1978). In the latter study they proved that the repetitive electrical stimulation of a node of Auerbach's plexus mainly produced an excitatory effect on the longitudinal muscle above, and an inhibitory effect below the stimulated spot, and they could support the concept of the law of the intestine which was postulated by BAYLISS and STARLING (1899). To understand the peristaltic reflex it seems to be necessary to clarify the properties of mechano- and chemosensitive neurons which are situated on the afferent pathways of the reflex arc within Auerbach's plexus. In the previous study (YOKOYAMA and OZAKI, 1978) it was reported that spontaneous neuronal discharges were occasionally recorded when the recording glass electrode was placed on the localized spot of a node of Auerbach's plexus. This paper deals with the effects of gut distension on activities of neutons in Auerbach's plexus as well as on the intestinal movements.

\section{METHODS}

Figure 1 shows the experimental arrangement. A 5-cm segment was excised from the small intestine of an adult rabbit anesthetized by a subcutaneous injec-

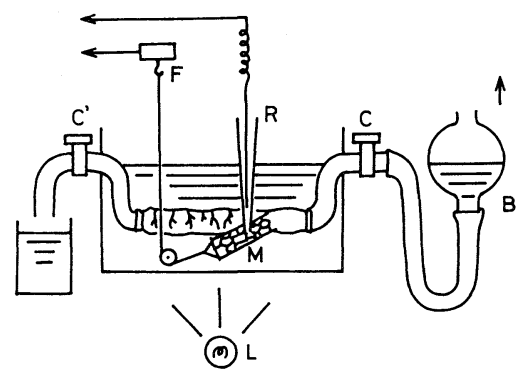

Fig. 1. Schematic representation of experimental arrangement. M, peeled longitudinal muscle strip which is attached to intestinal segment. $\mathrm{R}$, glass electrode placed on a node. L, light. $\mathrm{F}$, force displacement transducer. $\mathrm{B}$, pressure bottle. $\mathrm{C}$ and $\mathrm{C}^{\prime}$, two-way stopcock at inflow and otuflow. 
tion of urethane $(1 \mathrm{~g} / \mathrm{kg}$ body $\mathrm{wt})$. The longitudinal muscle strip (M) with adherent Auerbach's plexus ( $2 \mathrm{~cm}$ in length and $0.5 \mathrm{~cm}$ in width) was peeled from this intestinal segment, one end of the peeled longitudinal muscle strip was left attached to the intestinal segment and the other end was free. The intestinal segment was arranged horizontally, following principally TRENDELENBURG's method (1917), in a 70-ml bath of Krebs-Henseleit solution of the following composition (mM): $\mathrm{NaCl}, 118.0 ; \mathrm{KCl}, 4.7 ; \mathrm{CaCl}_{2} \cdot 2 \mathrm{H}_{2} \mathrm{O}, 2.5 ; \mathrm{MgSO}_{4} \cdot 7 \mathrm{H}_{2} \mathrm{O}, 1.2 ; \mathrm{KH}_{2} \mathrm{PO}_{4}$, 1.0; $\mathrm{NaHCO}_{3}, 25.0$; glucose, 5.5. The solution was aerated with $95 \% \mathrm{O}_{2}-5 \%$ $\mathrm{CO}_{2}$ and kept at $38^{\circ} \mathrm{C}$. The $\mathrm{pH}$ of the solution was 7.4-7.5. In general, the oral end of the segment was tied to the outflow tube, the anal end was tied to the inflow tube, and in several examples vice versa. Gut distension was realized by elevation of the pressure bottle (B), a two-way stop cock at the inflow (C) being opened and another cock at the outflow $\left(\mathrm{C}^{\prime}\right)$ shut.

For recording of movements of the peeled longitudinal muscle strip, the free end of this strip was connected to the force displacement transducer ( $F$, Grass FT 03), Another end of the muscle strip was left attached to the intestinal segment very near the inflow tube, as is shown in Fig. 1, so that the movements of the intestinal segment did not influence those of the peeled longitudinal muscle strip. The longitudinal muscle strip performed rhythmic auxotonic contractions. The muscle fibers and the nerve plexus of the peeled longitudinal muscle strip were connected with those of the intestinal segment.

The longitudinal muscle strip was transilluminated from below by a light (L) and Auerbach's plexus lying on the longitudinal muscle sheet could easily be seen under a stereodissecting microscope providing a range of magnification from 16 to $80 \times$. Care was taken that there was no notable damage of the network of Auerbach's plexus in the longitudinal muscle strip. Preparations with a partial break or lack of network of Auerbach's plexus were abandoned.

The glass electrode $(\mathrm{R})$ which was filled with Krebs-Henseleit solution and whose tip diameter was $20-30 \mu \mathrm{m}$ was placed on a local spot of a node of Auerbach's plexus. This electrode was mounted flexibly on the longitudinal muscle strip in the manner described by WoODBURY and BRAY (1956). Thus, the glass electrode placed on a node enabled recording of both muscle action potentials and spontaneous discharges of neurons in Auerbach's plexus. For recording electrical activities of both muscle fibers and neurons, muscle movement, a cathode ray oscilloscope (Nihon-Kohden Model VC-9) was used. In several examples, a spike counter (Diamedical system Model DSE-322) was used for the record of frequencies of spontaneous discharges of neurons. In such cases, spike potentials of neuron with an amplitude of $25-80 \mu \mathrm{V}$ were calculated and muscle action potentials larger than $90 \mu \mathrm{V}$ were eliminated. The frequencies of neuronal discharges in each second were recorded as sawtooth waves. Thus, the mixing of small muscle action potentials of less than $80 \mu \mathrm{V}$ in calculation could not be avoided. 


\section{RESULTS}

At first the behaviors of the longitudinal muscle and neurons in Auerbach's plexus were investigated at zero pressure on the intestinal segment. When the glass electrode was placed on a local spot of a node, action potentials of both longitudinal muscle and neuron could be recorded. In general, 5 or $10 \mathrm{~min}$ after soaking the preparation in the solution, rhythmic bursts of muscle action potentials began to appear. Each burst consisted of 2-5 spike potentials (Figs. 2B, $3 \mathrm{~A}, 5 \mathrm{~b}, 6 \mathrm{~A}, 7 \mathrm{~A}$ ) with amplitudes in the range of $0.05-1.0 \mathrm{mV}$. Twenty or $30 \mathrm{~min}$ after soaking the preparations, contractions of intestinal muscle became in general regularly rhythmic. However, contractions of duodenal preparations were irregular for a long time (more than 1 or $2 \mathrm{hr}$ ). Therefore, in the present study mostly jejunal and ileal preparations were used. Intervals of bursts of muscle action potentials had the value of 3-5 sec which was in accordance with rhythmic contractions of both the longitudinal muscle strip and the intestinal segment. The contractions of the intestinal segment were of a pendular movement. Muscle action potentials in each burst appeared just before the onset of muscle contractions (Figs. 2B, 3A, 5b, 6A, 7A).

\section{Spontaneous discharges of neurons}

Twenty or $30 \mathrm{~min}$ after soaking the preparation in the bath solution, spontaneous discharges of neuron could be recorded by the glass electrode placed on a node. Figure 2A illustrates various spots of nodes of Auerbach's plexus from which various types of pattern of spontaneous neuronal discharges could be obtained (Fig. 2B).

Frequent spike discharges whose frequency and amplitude were irregular and not uniform, respectively, were obtained from spots, 1,5 and 7. These irregular spontaneous discharges may signify the activities of multiunit neurons. Uniform spontaneous discharges with spike height unchanged were obtained from spots 2 , $3,4,6,8,9,11$ and 12 . Tracings 2 and 3 showed biphasic action potentials, tracings $4,9,11$ showed negative potentials, tracings $6,8,12$ showed positive potentials. It was remarkable that in tracings $2,4,6,8,9,11$, frequencies of neuronal discharges decreased during the phase of muscle relaxation (upward deflexion in the lower tracing indicates relaxation) and increased during the phase of contraction. In tracing 12, neuronal discharges showed variations in frequencies but there was no correlation between frequency change and the phase of muscle contractions. In tracing 3 neuronal discharges were characterized by periodic activities consisting of groups of spike bursts separated by periods of silence. In this tracing frequencies of neuronal discharges in bursts had no correlation with the phase of muscle contractions. In tracing 10, only muscle action potentials were recorded and there was no neuronal discharge. When the glass electrode was placed on the nerve bundle between nodes, neuronal discharges could be, but not in all cases, obtained which mostly showed irregular spike dis- 


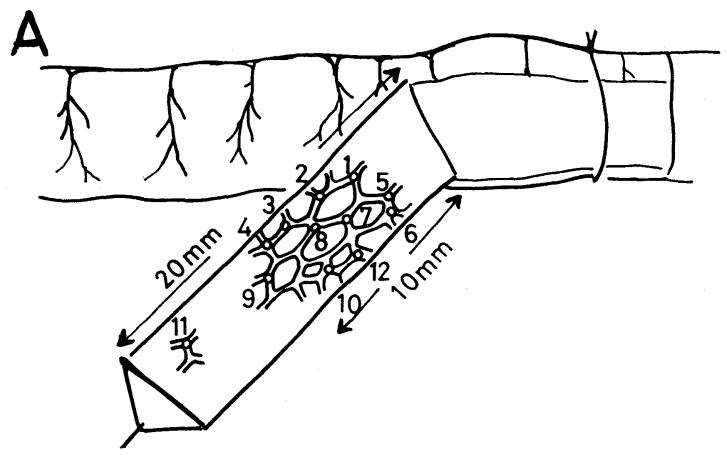

B

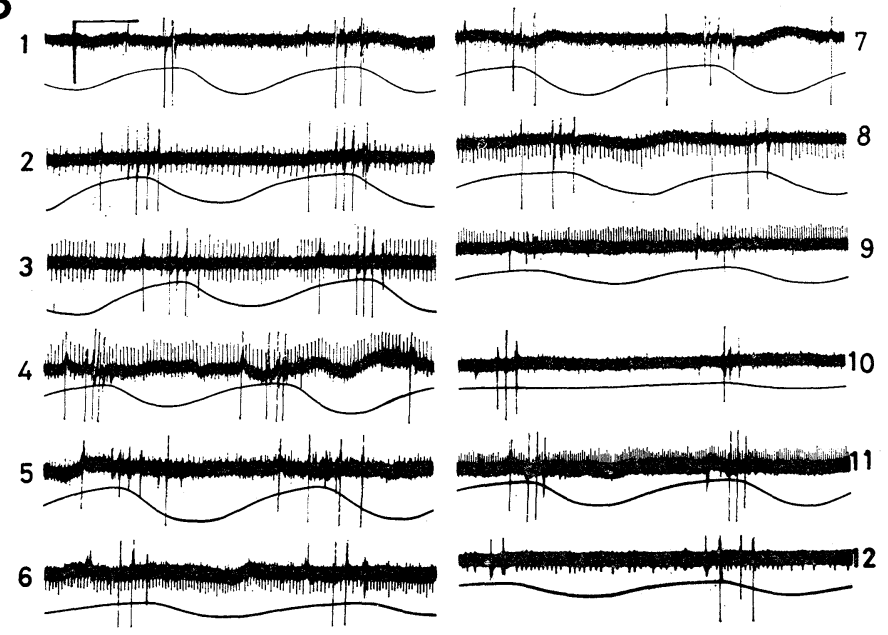

Fig. 2. A: Schematic drawing of network of Auerbach's plexus in the peeled longitudinal muscle strip which is attached to intestinal segment. Numbers 1-12 indicate spots on nodes where spontaneous muscular and neuronal discharges were led. B: Recording of electrical activities of muscle and neuron (upper tracing), muscle contractions (lower tracing, upward deflexion shows relaxation). Numbers 1-12 indicate tracings which were obtained from spots illustrated in A. Calibrations: $100 \mu \mathrm{V}$, tension development in $1 \mathrm{~g}$, and $1 \mathrm{sec}$.

charges as shown in tracings 1, 5, 7. Amplitude of uniform neuronal discharges was generally in the range of $30-80 \mu \mathrm{V}$ and duration of each spike had the value of 2.0-2.5 msec. Although spike amplitude of neuronal discharges varied according to spots, spike amplitude in each tracing $(2,3,4,6,8,9,11,12)$ remained almost the same. Such uniform spike discharges may signify the activities of a single unit neuron.

That the above-described spontaneous discharges of small spikes are due to the activation of neurons can be concluded from the following 3 observations. The first is that these spontaneous discharges could not be obtained when the 
electrode was placed on the longitudinal muscle layer outside the network of Auerbach's plexus. In this case only muscle action potentials were recorded. The second is that some of these spontaneous discharages responded to the distension of the intestinal segment with exictation or inhibition. The third is that all these spontaneous discharges were abolished 1 or 2 min after application of tetrodotoxin $\left(1 \times 10^{-7} \mathrm{~g} / \mathrm{ml}\right)$, while muscle action potentials as well as movements of the peeled longitudinal muscle and the intestinal segment were not affected by tetrodotoxin (not shown, unpublished data).

In the present study, 164 preparations (154 jejunal, 10 ileal) taken from 68 rabbits were used and 292 spots of nodes were examined. In these spots, 159 showed irregular spontaneous neuronal discharges, 96 showed uniform discharges and 37 did not show any neuronal discharges. One type of neuronal discharges generally continued for a long time, namely $0.5-1.5 \mathrm{hr}$. However, the conversion of one type into another occasionally occurred, irregular spike discharges were converted into uniform ones and vice versa.

\section{Effects of gut distension}

When the intraluminal pressure was raised from zero to $5 \mathrm{~cm} \mathrm{H}_{2} \mathrm{O}$ in about $2 \mathrm{sec}$, some spontaneous neuronal discharges responded with excitation, other responded with inhibition and some others showed no response. Figure 3 shows an example of the excitatory effect of gut distension on neuronal activities. Spontaneous neuronal discharges appeared at the control period of zero pressure with the same spike amplitude and characteristic changes of spike frequency. Their frequencies were $13-18 \mathrm{~Hz}$ in the phase of muscle relaxation and they increased in the phase of muscle contraction up to $20-22 \mathrm{~Hz}$ (Fig. 3A, a, upward deflexion in the lower curve shows relaxation; lower tracing in Fig. 3B). Immediately after elevation of the intraluminal pressure to $5 \mathrm{~cm} \mathrm{H}_{2} \mathrm{O}$ the frequency of spontaneous discharges began to increase, reaching a maximal value of $30 \mathrm{~Hz}$. The increased frequency continued during maintained distension of about a 20 -sec period (Fig. 3A, b-d; lower tracing in Fig. 3B) and decreased rapidly by lowering the pressure to zero. Such an excitatory effect of gut distension on uniform discharges was obtained in 72 of 96 examples of uniform neuronal discharges. In accordance with the appearance of frequency increase of neuronal discharges, intervals of contractions of the longitudinal muscle strip as well as of the intestinal segment were shortened (Fig. 3A, b-d; upper tracing in Fig. 3B, from 8th interval to 12 th). On inspection it was found that all movements of the intestinal segment became peristaltic during the period of distension, contraction waves always traveled from the oral end of the intestinal segemnt to the aboral end. In all examples of experiments of gut distension, an excitatory effect on the longitudinal muscle as well as on the intestinal segment was observed.

Using a spike counter, frequencies of uniform neuronal discharges were recorded as sawtooth waves and the effects of gut distension were studied. Figure $4 \mathrm{~A}$ shows an example. In the control period at an intraluminal pressure of zero, 


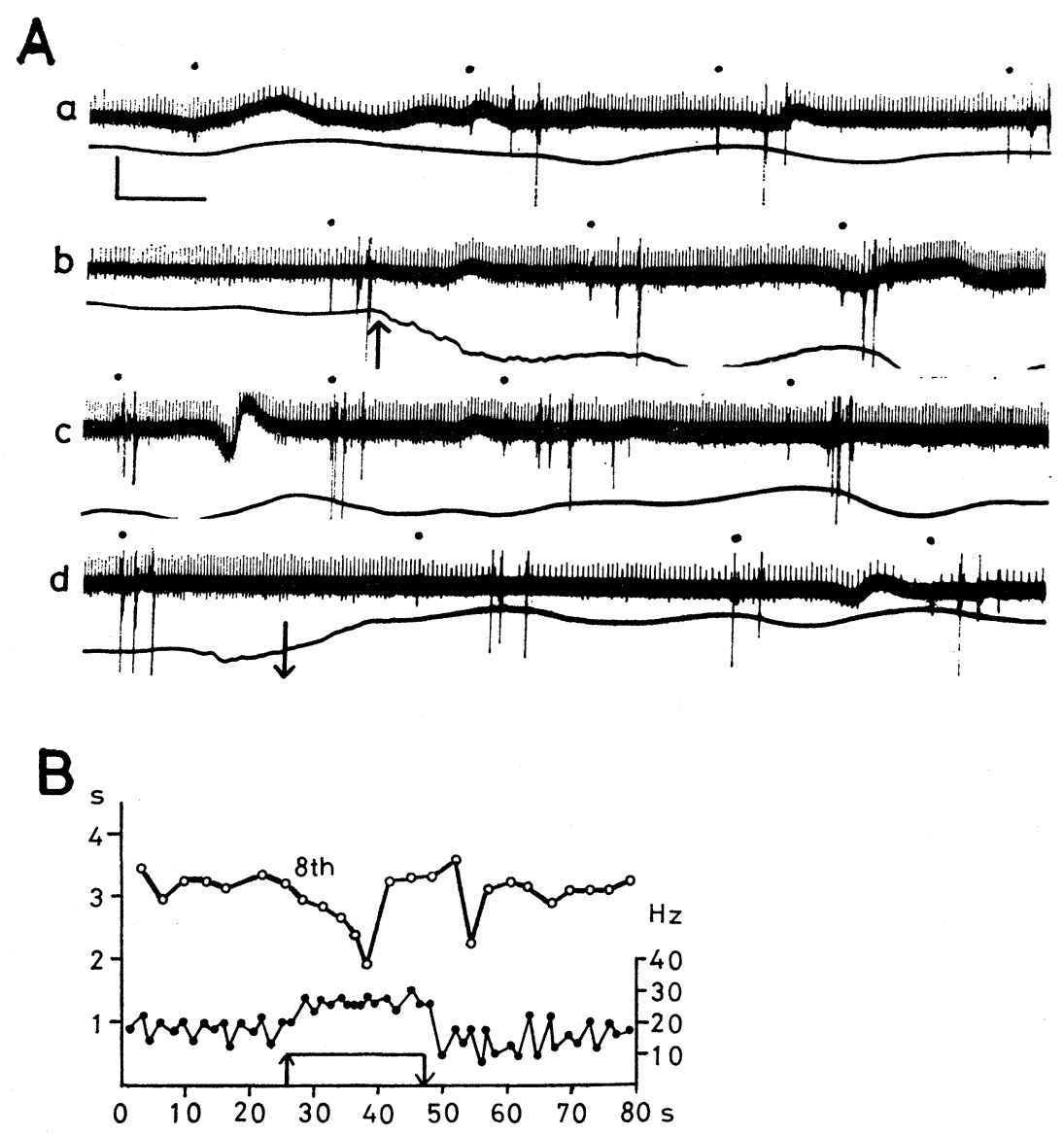

Fig. 3. Excitatory effects of gut distension on muscular and neuronal activities. A: Upper tracings show muscular (marked with $\bullet$ ) and neuronal discharges whose frequency decreased during muscle relaxation and increased during muscle contraction (a). Lower tracings show muscle contraction (upward deflexion indicates relaxation). Arrows indicate the period of elevation of intraluminal pressure to $5 \mathrm{~cm} \mathrm{H}_{2} \mathrm{O}$ (b-d). Calibrations: $50 \mu \mathrm{V}$, tension change in $0.5 \mathrm{~g}$, and $1 \mathrm{sec}$. B: Graphic representation of changes of intervals of muscle contractions (upper curve, open circles) and frequencies of neuronal discharges (lower curve, filled circles), obtained from the experiment shown in A. Arrows indicate the period of gut distension. Shortening of intervals of muscle contractions began from the 8th interval. Frequencies of neuronal discharges were measured at both phases of relaxation and contraction. Ordinate at left side: intervals of muscle contraction in sec; that at right side: frequency of neuronal discharges. Abscissa, time course in sec.

frequencies of neuronal discharges showed wave-like variations, being increased to $16 \mathrm{~Hz}$ during the phase of muscle contraction and being decreased to $7 \mathrm{~Hz}$ during the phase of relaxation (Fig. 4A, a). By elevation of intraluminal pres- 
A
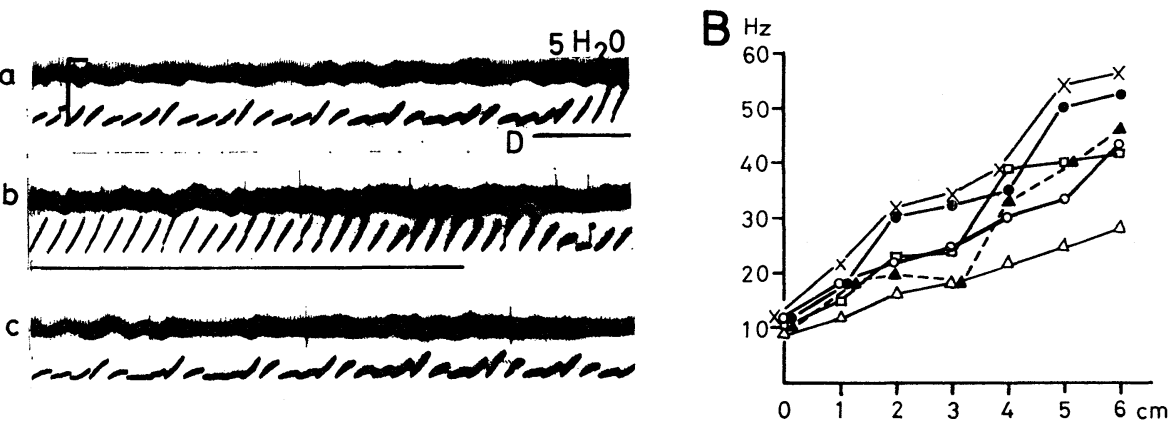

Fig. 4. Excitatory effect of gut distension on neuronal discharges. A: Continuous recording of neuronal discharges (upper tracing) and spike frequencies of neuronal discharges recorded by a spike counter (lower tracing, sawtooth-like waves). D indicates the beginning of distenison. Straight line under tracings indicates the period of elevation of intraluminal pressure to $5 \mathrm{~cm} \mathrm{H}_{2} \mathrm{O}(\mathrm{a}-\mathrm{b})$. Calibrations: $100 \mu \mathrm{V}$, frequency of $50 \mathrm{~Hz}$, and $1 \mathrm{sec}$. B: Relationship between frequencies of neuronal discharges and intraluminal pressures, obtained from 6 experiments. Ordinate, frequency of neuronal discharges. Abscissa, intraluminal pressure in $\mathrm{cm}_{2} \mathrm{O}$.

sure to $5 \mathrm{~cm} \mathrm{H}_{2} \mathrm{O}$, frequencies of neuronal discharges increased up to $33 \mathrm{~Hz}$ (Fig. 4A, a-b) and by lowering the pressure to zero, frequencies of discharges decreased and again showed wave-like variations (Fig. 4A, c). In 6 experiments the relationship between the frequencies of uniform neuronal discharges and the intraluminal pressures was plotted (Fig. 4B). In this diagram, the mean values of frequencies during the control zero pressure period of $20-30 \mathrm{sec}$ were used and the maximal frequencies which were obtained during the period of gut distension were used as the frequencies corresponding to the pressures. It was ascertained that the uniform neuronal discharges whose frequencies were increased during muscle contraction and decreased during muscle relaxation, as shown in tracings 2, 4, 6, 8, 9, 11 of Fig. 2B and in Fig. 3A, were related to the intensity of gut distension; the higher the intraluminal pressure, the more frequent was the neuronal discharge.

Figure $5 \mathrm{~b}$ shows the excitatory effect of gut distension on irregular neuronal activities. By elevation of intraluminal pressure from zero to $5 \mathrm{~cm} \mathrm{H}_{2} \mathrm{O}$, the amplitude of burst of neuronal discharges was increased and decreased after the lowering of intraluminal pressure to zero. By using a spike counter it was ascertained that the frequency of neuronal discharges also increased during the period of gut distension. In one experiment the value of $45 \mathrm{~Hz}$ at the control period of zero pressure was increased up to $110 \mathrm{~Hz}$ during the period of elevation of intraluminal pressure to $5 \mathrm{~cm} \mathrm{H}_{2} \mathrm{O}$. Such an excitatory effect was observed in 131 of 159 examples of irregular neuronal discharges. In 28 other examples there was no marked change in frequency of neuronal discharges by gut distension. 


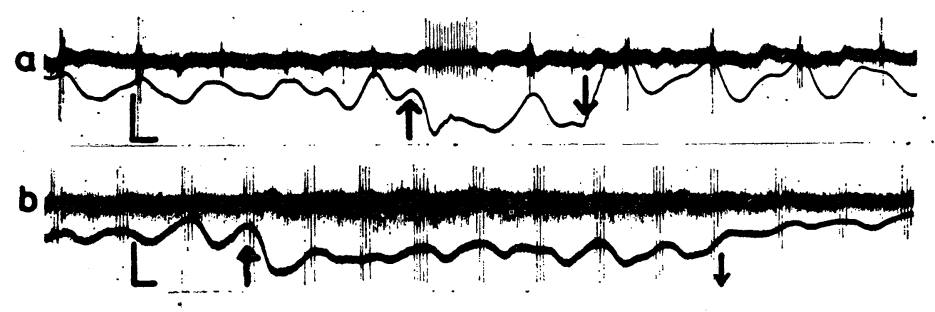

Fig. 5. a: Rapidly adapting phasic-type response of nueron. Upper tracing shows electrical activities of both longitudinal muscle and neuron. Lower tracing, muscle contractions (upward deflexion indicates muscle relaxation). Arrows indicate the period of elevation of intraluminal pressure to $5 \mathrm{~cm} \mathrm{H}_{2} \mathrm{O}$. b: Effects of gut distension on irregular neuronal discharges and muscle contractions. Explanations are same as in a. Calibrations: $50 \mu \mathrm{V}$, tension change in $0.5 \mathrm{~g}$, and $1 \mathrm{sec}$.

Figure 5a shows an example of the evoked excitation of a single neuron by gut distension. In this case neuronal discharges could not be recorded at zero intraluminal pressure. Immediately after elevation of intraluminal pressure to $5 \mathrm{~cm} \mathrm{H} \mathrm{H}_{2} \mathrm{O}$, uniform regular spike potentials were evoked which continued for several seconds and disappeared during the period of gut distension. Such a rapidly adapting response was observed only in 5 of 37 cases in which spontaneous discharges were not observed at the intraluminal pressure of zero. In general, the amplitude of evoked spike potentials was large $(50-70 \mu \mathrm{V})$.

Figure 6 shows an example of the inhibitory effect of gut distension upon neuronal discharges. Uniform neuronal discharges were recorded at the control period of zero intraluminal pressure. Frequencies of spontaneous discharges varied in time course. However, there was no correlation between frequencies of neuronal discharges and the phase of muscle contractions, as was illustrated in tracing 12 of Fig. 2B and shown diagramatically in the lower curve of Fig. 6B. These spontaneous discharges disappeared several sceonds after the elevation of intraluminal pressure to $5 \mathrm{~cm} \mathrm{H}_{2} \mathrm{O}$ (Fig. 6A, b-c; lower curve in Fig. 6B). During the period of distension spontaneous discharges again appeared (Fig. 6A, c; lower curve in Fig. 6B) which continued after the lowering of intraluminal pressure to zero and also showed no correlation in their frequencies with the phase of muscle contractions (Fig. 6A, d; lower curve of Fig. 6B). Although the inhibitory effect occurred in neuronal activities, a notable shortening of intervals of muscle contractions appeared during the phase of gut distension (Fig. 6A, c; upper curve in Fig. 6B). The inhibitory effect of gut distension on neuronal activities was observed in 11 of 96 examples of uniform neuronal discharges. In general, the amplitude of neuronal discharges of this type neuron was small $(30-40 \mu \mathrm{V})$.

Figure 7 shows an example of no effect of gut distension. Uniform neuronal discharges showed periodic activities consisting of groups of spike bursts separated by silence. Frequencies of neuronal discharges in each burst had no correlation with the phase of muscle contractions (Fig. 7A, a; lower tracing in Fig. 7B). By 

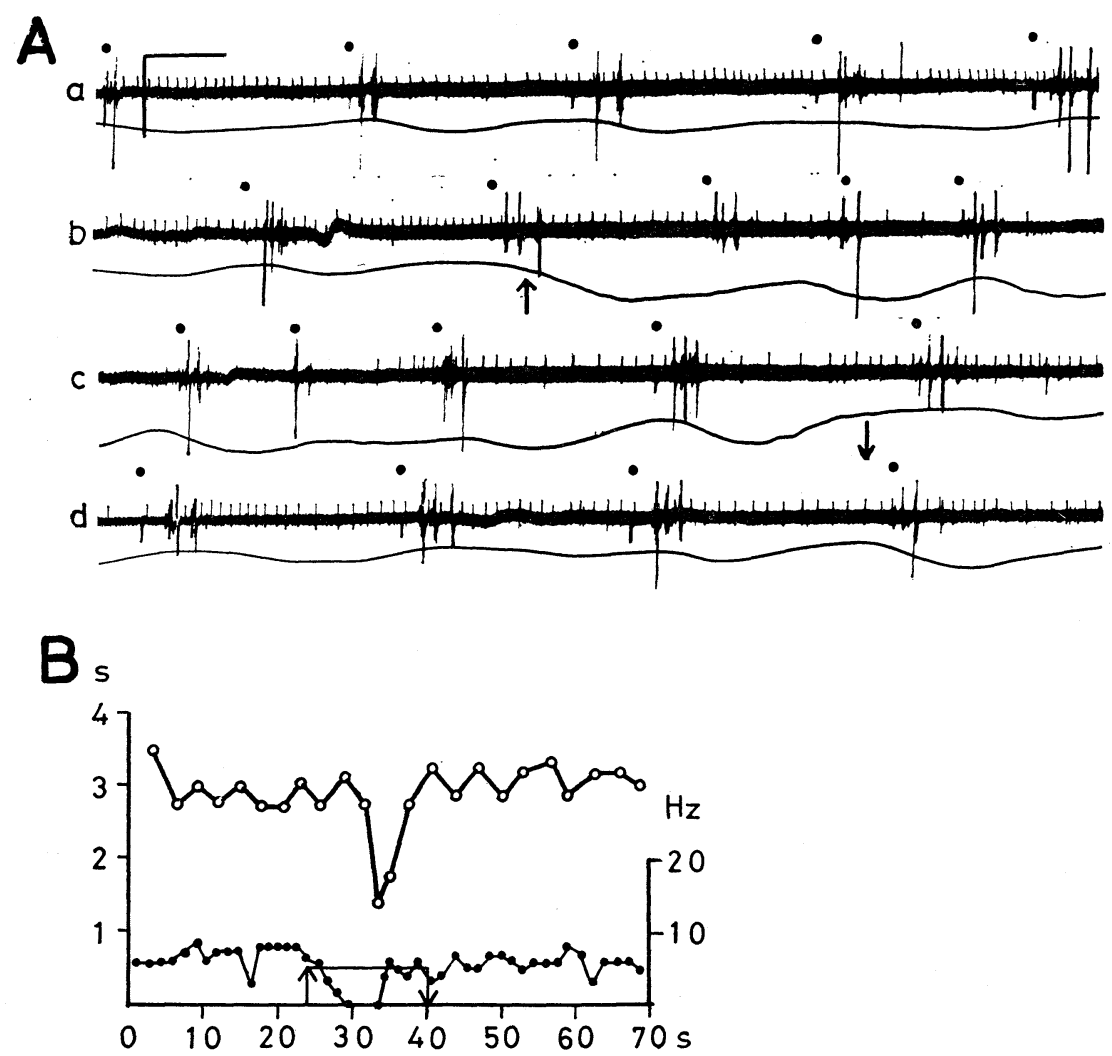

Fig. 6. Inhibitory effect of gut distension on neuronal discharges. A: Upper tracings show muscular (marked with $\bullet$ ) and neuronal discharges whose frequency did not relate to phase of muscle contractions (upward deflexion shows relaxation). Arrows indicate the period of elevation of intraluminal pressure to $5 \mathrm{~cm} \mathrm{H}_{2} \mathrm{O}(\mathrm{b}-\mathrm{c})$. Calibrations: $100 \mu \mathrm{V}$, tension change in $1.0 \mathrm{~g}$, and $1 \mathrm{sec}$. B: Graphic representation of changes of intervals of muscle contractions (upper curve, open circles) and frequencies of neuronal discharges (lower curve, filled circles), obtained from the experiment shown in A. Arrows indicate the period of gut distension. Ordinate at left side: intervals of muscle contractions in sec; that at right side: frequency of neuronal discharges. Abscissa, time course in sec.

elevation of intraluminal pressure to $5 \mathrm{~cm} \mathrm{H}_{2} \mathrm{O}$, shortening of intervals of muscle contractions and groups of neuronal spike bursts occurred but there was no change in spike frequency of neuronal discharges (Fig. 7A, b-c; Fig. 7B). It is thought that there are some neurons in Auerbach's plexus which discharge spontaneously, being accompanied by silence, but do not respond to gut distension. The amplitude of discharges of this type neuron was in general large (60-70 $\mu \mathrm{V})$. No such effect of gut distension on neuronal activities was observed in 13 of 96 cases of uniform neuronal discharges. 

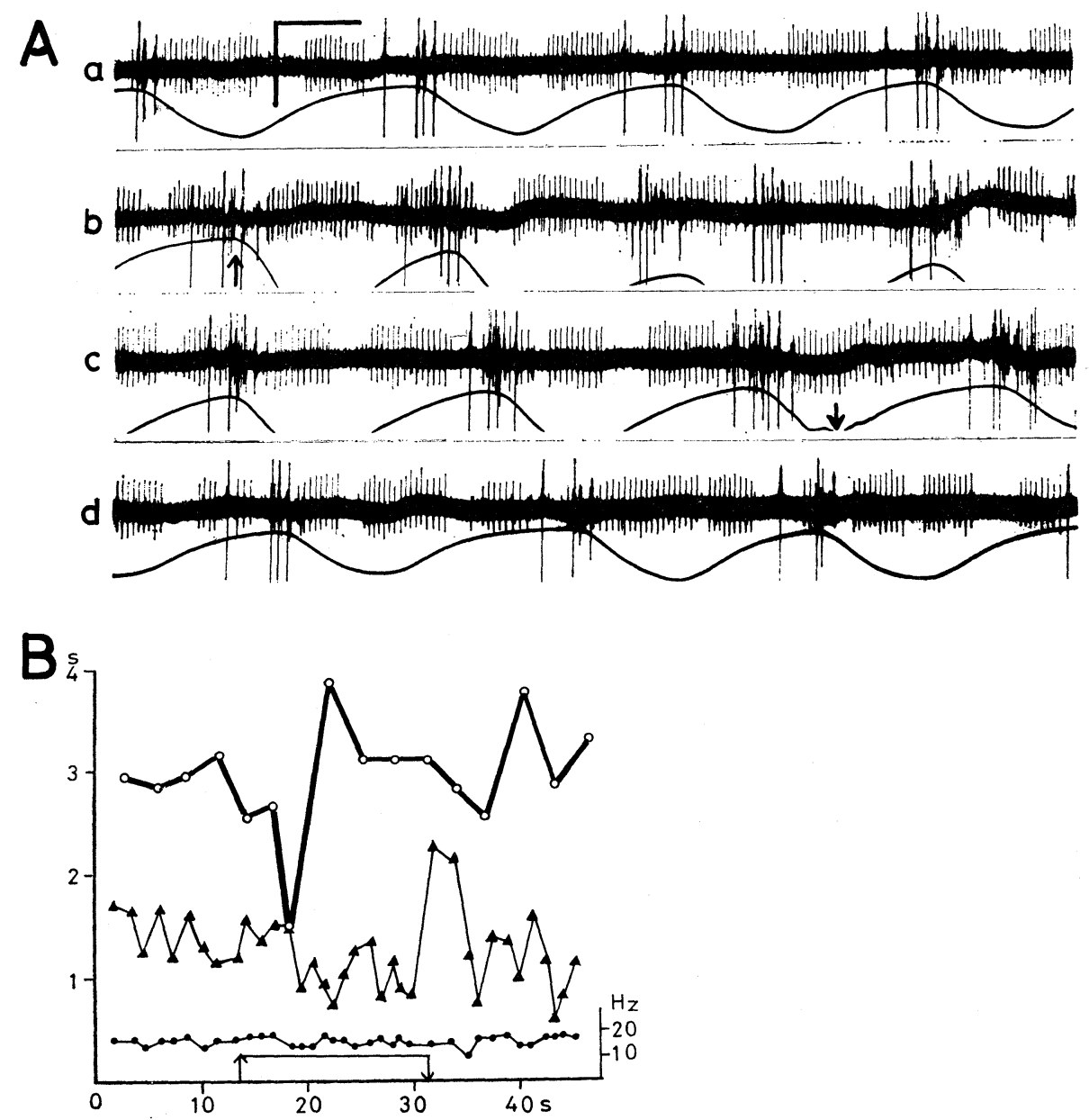

Fig. 7. No effect of gut distension upon neuronal discharges which appeared as groups of spike bursts separated by periods of silence. A: Continuous recording of electrical activities of muscle and neuron (upper tracings) and muscle contractions (lower tracing, upward deflexion shows relaxation). Explanation and calibrations are the same as in Fig. 6A. B: Graphic representation of intervals of muscle contractions (upper curve, open cirlces), of groups of spike bursts (middle curve, triangles) and frequency of neuronal discharges (lower curve, filled circles). Explanations are the same as in Fig. 6B.

\section{Effects of muscle stretching on spontaneous neuronal discharges}

From the above-described results it may be thought that there is a mechanosensitive receptor in the wall of the rabbit small intestine and this receptor activates a specific neuron in Auerbach's plexus when the intestinal segment is distended. The question arises whether this mechanosensitive receptor is located in the muscle layers and activated by stretching the intestinal muscle in the longitudinal direction or circular direction. 


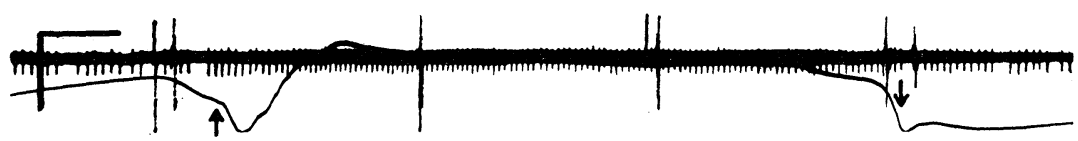

Fig. 8. Excitatory effect of stretching the longitudinal muscle in the longitudinal direction on neuronal discharges. Upper tracing shows electrical activities of muscle and neuron. Lower curve shows muscle contraction (upward deflexion indicates tension development). Arrows indicate the period of stretching. Calibrations: $100 \mu \mathrm{V}$, tension development in $1 \mathrm{~g}$ and $1 \mathrm{sec}$.

Figure 8 illustrates one example of the exitatory effect of stretching the longitudinal muscle in the longitudinal direction upon neuronal discharge. Stretch stimulus was given by elevating the transducer ( $\mathrm{F}$, Fig. 1 ), namely by pulling the peeled longitudinal muscle in the longitudinal direction. Spontaneous neuronal discharges showed in this preparation a frequency increase during muscle contraction $(12 \mathrm{~Hz})$ and a frequency decrease during muscle relaxation $(8 \mathrm{~Hz})$. Immediately after tension development (upward deflexion) of the longitudinal muscle strip, the frequency increased to about $16 \mathrm{~Hz}$, the increased frequency continued during the period of tension development and decreased after the reduction of tension. The rate of frequency increase by stretching the longitudinal muscle in the longitudinal direction was in the range of 1.5-1.7 which was observed in 5 cases. Thus, it may be thought that the mechanosensitive receptors are arranged in series with the longitudinal muscle, as IGGo $(1955,1957)$ postulated.

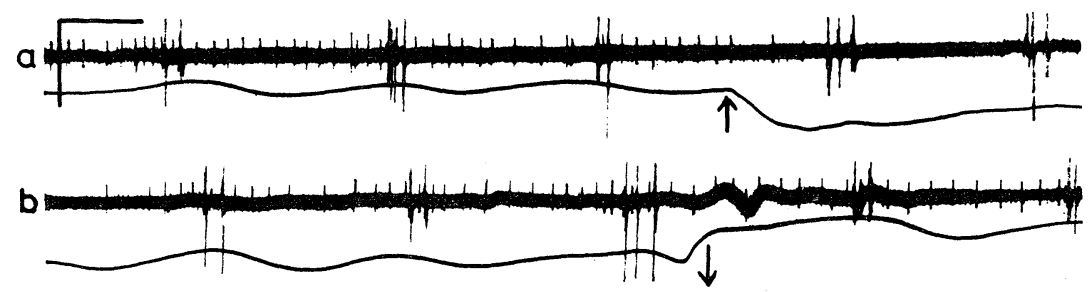

Fig. 9. Inhibitory effect of stretching the longitudinal muscle in the longitudinal direction on neuronal discharges. Upper tracing shows electrical activities of muscle and neuron. Lower tracing shows muscle contractions (downward deflexion indicates tension development). Arrows indicate the period of stretching. Calibrations: $100 \mu \mathrm{V}$, tension change in $1 \mathrm{~g}$, and $1 \mathrm{sec}$.

Figure 9 shows an example of the inhibitory effect of stretching the longitudinal muscle in the longitudinal direction upon neuronal activities. In this example, the frequency of neuronal discharge showed no correlation with the phase of muscle contraction, and the inhibitory effect of gut distension was observed, as is illustrated in Fig. 6. By longitudinal stretching of the longitudinal muscle, neuronal discharges disappeared for several seconds (Fig. 9a) and during stretching neuronal discharges again appeared (Fig. 9b). Such an inhibitory effect was observed in 3 of 4 examples examined. 


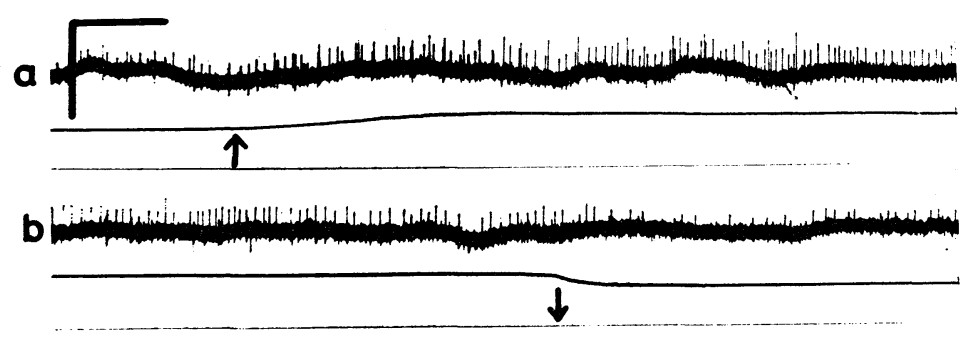

Fig. 10. Excitatory effect of stretching the longitudinal muscle strip in the circular direction. Upper tracing shows electrical activity of neuron. Lower curve shows tension change by stretching the muscle strip in circular direction (upward deflexion indicates tension development). Arrows indicate the period of stretching. Calibrations: $100 \mu \mathrm{V}$, tension development in $1 \mathrm{~g}$, and $1 \mathrm{sec}$.

Figure 10 shows an example of the excitatory effect of stretching the longitudinal muscle in the circular direction. The stretch stimulus in the circular direction was given to the peeled longitudinal muscle which was suspended horizontally in the bath, as described in the previous study of YoKOYAMA et al. (1977). The frequency increase of supontaneous neuronal discharges was observed during the period of muscle stretching in the circular direction. Such an excitatory effect was observed in 4 or 5 cases examined. The rate of frequency increase was in the range of $1.3-1.5$.

\section{Extent of the effect of gut distension}

Excitatory and inhibitory effects of gut distension on spontaneous neuronal discharges generally were obtained when the examined nodes were situated within $10 \mathrm{~mm}$ measured from the junction of the longitudinal muscle strip and the intestinal segment, as is illustrated in Fig. 2A. When the examined node was situated $19 \mathrm{~mm}$ from the juction (Fig. 2A, spot 11), an effect of gut distension on neuronal activities was not observed.

\section{Localization of stretch receptor}

The question arises whether or not the mucosa participates in the excitatory effects of gut distension on both neuronal and muscular activities. The effect of removal of mucosa was examined. At first the intestinal segment was turned inside out. With a glass plate the whole mucosa, including muscularis mucosa and Meissner's plexus, was scraped off, then the intestinal segment consisting of both longitudinal and ciruclar muscles was returned to its normal position. The obtained intestinal segment with the peeled longitudinal muscle strip was arranged as shown in Fig. 1. Figure 11 shows an example of the effects of gut distension. Spontaneous neuronal discharges were observed at zero intraluminal pressure and were irregular both in spike amplitude and in frequency. Amplitude of neuronal discharges was increased by elevation of the intraluminal pressure to $5 \mathrm{~cm} \mathrm{H}_{2} \mathrm{O}$ and prominent shortening of intervals of muscle spike burst appeared. 


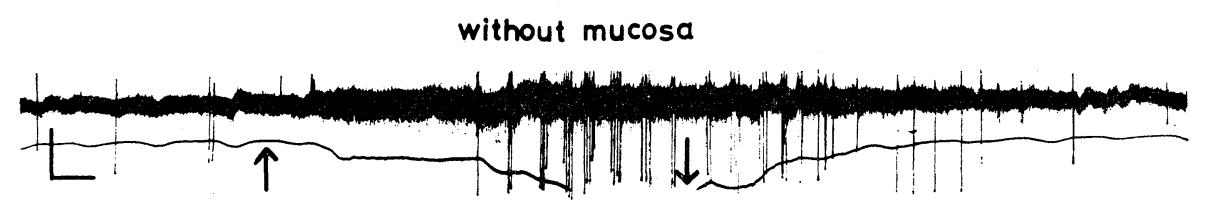

Fig. 11. Excitatory effect of gut distension on neuronal discharges and muscle contractions. Mucosa of the intestinal segment was removed previously. Upper tracing shows muscular and neuronal discharges. Neuronal discharges appeared as spike bursts in which amplitude and frequency of spikes were irregular. Lower tracing shows change of muscle tension (downward deflexion shows tension development). Arrows indicate the period of gut distension. Calibrations: $50 \mu \mathrm{V}$, tension development in $0.5 \mathrm{~g}$, and $2 \mathrm{sec}$.

On inspection, strong propulsive contractions of the intestinal segment (peristaltic movements) were observed and these contractions continued not only during the period of gut distension but also for $20 \mathrm{sec}$ after lowering of the pressure to zero. In 4 other experiments these notable excitatory effects of gut distension on both neuronal activities and intestinal muscles were observed. On all 5 preparations used in this series of experiments, methylene blue staining was done after the experiment following SCHABADASCH's method (1930) and it was ascertained under a microscope that mucous membrane including Meissner's plexus was removed entirely and only longitudinal and circular muscle layers including Auerbach's plexus remained. From these results it can be concluded that the stretch receptors which activate neuronal activities by gut distension and cause the excitation of intestinal muscles are mainly located in the muscularis externa.

\section{Polarity of effects of gut distension on peeled longitudinal muscle}

In the previous study (YoKoYAMA and OzAKI, 1978), the polarity of effects of stimulation of Auerbach's plexus on the longitudinal muscle was proved. It was shown in this study that repetitive electrical stimulation of a local spot of a node mainly caused excitation of the orally situated longitudinal muscle and inhibition of that part anally situated. The question arises whether or not the distension effect on the longitudinal muscle strip varies according to the junction of the muscle strip with the intestinal segment. When the oral part of the intestinal segment was connected to the ourflow tube (at left side in Fig. 1), the longitudinal muscle strip was attached to the anal end of the intestinal segment, and vice versa. From many observations in the present study, it was noticed that there was no marked difference in the effects of gut distension on the longitudinal muscle strip according to whether the junction of the muscle strip was at anal or oral part of the intestinal segment. It is thought that, since many nerve cells in Auerbach's plexus to a large degree were excited almost simultaneously by a rapid inflow of the solution into the intestinal segment, the polarity of distension effects on the longitudinal muscle strip could not be produced. 


\section{DISCUSSION}

From the above-described results, neurons within Auerbach's plexus may be classified into two main categories. The first category consists of mechanosensitive neurons and the second consists of neurons whose activities are not changed by gut distension.

There are several types of mechanosensitive neurons. The first type showed spontaneous discharges whose frequencies increased during muscle contraction and decreased during muscle relaxation (tracings 2, 4, 6, 8, 9, 11 in Fig. 2B; Figs. 3A, 4A) and which responded to gut distension with excitation (Figs. 3A, 4A). Since a neuron of this type could also be activated by stretching the longitudinal muscle in the longitudinal direction (Fig. 8), the stretch receptors in the intestinal wall may be arranged in series with longitudinal muscle fibers, as was postulated by IGGo $(1955,1957)$. The excitatory effect produced on this type of neuron by gut distension continued during, and several seconds after the end of gut distension. Thus, this neuron is of the slowly-adapting phasic-type which was reported by Wood (1970), Wood and MAYer (1974). Hirst and McKirdy (1974), using the method of intracellular recording of neuron action potentials, observed a frequency increase of synaptic potentials which was produced by gut distension at the oral side of guinea pig ileum. This neuron may also belong to the slowlyadapting phasic-type. In the present study the neurons of slowly-adapting phasictype were found in a large number ( 72 of 96 cases of uniform spontaneous discharges, 131 of 159 cases of irregular ones).

The second type of mechanosensitive neurons was silent under zero intraluminal pressure and responded to gut distension with a burst of spike potentials which continued only at the beginning of gut distension (Fig. 5a). Such fastadapting phasic-type neurons were found in the present study in a small number (5 of 37 silent cases).

The third type of mechanosensitive neurons is characterized by inhibition which was produced by gut distension (Fig. 6A). It is interesting that spontaneous discharges of this type of neuron had no correlation in frequency with the phase of muscle contractions (tracing 12 in Fig. 2B; Fig. 6A) and, in addition, activities of neurons of this type were inhibited by stretching the longitudinal muscle in the longitudinal direction (Fig. 9). This type of neuron was also found in a small number (11 of 96 cases of uniform spontaneous discharges).

The functions of the above-described mechanosensitive neurons are now obscure. From the studies of intracellular recording of neuron action potentials the existence of two distinct types of nerve cells in Auerbach's plexus was postulated (Nishi and North, 1973; Hirst et al., 1974; Hirst and McKirdy, 1974; Hirst et al., 1975). One type is characterized by a very long after-hyperpolarization and by not receiving synaptic input. This cell is called $\mathrm{AH}$ cell and is presumed to be an afferent nerve cell. Another type is characterized by receiving synaptic input and is called $\mathrm{S}$ cell and is supposed to be an internuncial or 
motor cell.

PainTal (1954) and IGGo $(1955,1957)$ investigated the functions of the distension-sensitive neurons in stomach and intestine of cat which send impulses to the central nervous system through the vagus nerve. Single $C$ fibers in the cervical vagus nerve which discharged spikes spontaneously showed a peak frequency of $35 \mathrm{~Hz}$ when the stomach or intestinal segment was distended with a rapid rate of inflow (IGGO, 1957). This value coincided approximately with values of increased frequency of neuronal discharges produced by elevation of the intraluminal pressure to $3-5 \mathrm{~cm} \mathrm{H}_{2} \mathrm{O}$ (Fig. $3 \mathrm{~B}$ and Fig. $4 \mathrm{~B}$ in the present study). It seems that some distension-sensitive neurons whose activities were shown in Fig. 3 and Fig. 4 may be afferent nerve cells.

The interneurons in Auerbach's plexus of guinea pig small intestine were suggested by HIRST and MCKIRDY (1974) and those in rabbit small intestine were postulated by YoKOYAMA and OzAKI (1978) from the results that the repetitive electrical stimulation of a localized spot of a node of Auerbach's plexus showed in many examples simultanous excitation of longitudinal muscle above, and inhibition below the stimulated spot. The existence of descending excitatory, as well as inhibitory neurons in Auerbach's plexus was suggested by HiRST et al. (1975), YoKOYAMA and OzAKI (1978), that of ascending excitatory neuron was postulated by YoKOYAMA and OZAKI (1978). Some distension-sensitive neurons as above described might be an interneuron in one part and in another part a motor neuron.

OHKAWA and Prosser (1972) reported the excitatory effect of gut distension on spontaneous neuronal discharges of cat small intestine. However, Wood (1970), OHKAWA and Prosser (1972) found no correlation between burst activity of neurons of Auerbach's plexus and activities of both longitudinal and circular muscles. In the present study spontaneous uniform discharges of many neurons showed wave-like variations in frequency (increased during muscle contraction, decreased during relaxation) and were excited by gut distension. In accordance with the increased frequency of neuronal discharges by gut distension, activities of both the longitudinal muscle strip and the intestinal segment were in all experiments excited. Activities of the distension-sensitive neuron in the peeled longitudinal muscle represent those of neurons in the wall of the intestinal segment. Therefore, it may be thought that the stretch receptors which were excited by gut distension cause the excitation of interneurons within Auerbach's plexus. The excited activities of interneurons affect the excitatory as well as the inhibitory neurons which regulate the intestinal muscle as a consequence of the integrity of their actions and cause peristaltic movement.

The function of the neurons within Auerbach's plexus which respond to gut distension or stretching of the longitudinal muscle with inhibition of neuronal discharges remains obscure.

The second category consists of neurons whose activity did not have any cor- 
relation with the phase of contractions of the intestinal muscle. Discharges of neurons of this type appeared as periodic groups of spike bursts separated by silence (tracing 3 in Fig. 2B; Fig. 7A). Spike frequency in bursts did not change by elevation of intraluminal pressure (Fig. 7). This type was found in a small number (11 of 96 uniform spontaneous discharges). The physiological function of the neuron of this type is also unclear.

IGGO (1957) reported that the distension-sensitive mechanoreceptors were unaffected by the removal of mucosa and submucosa, and probably lie in the muscularis externa of the stomach and intestine. In the present study it was proved that the function of mechanoreceptors which activated the specific neurons and caused an excitatory effect on intestinal muscle remained after scraping the mucosa and submucosa off. This result coincided with that of IGGo (1957). However, this result does not exclude the existence of mechanoreceptors and chemoreceptors in the mucosa which produce the peristaltic reflex by mechanical or chemical stimulation of the mucosa (HuKuHARA et al., 1958). BüLBRING et al. (1958) studied the peristaltic response of guinea pig ileum and found that the peristaltic reflex could be abolished by procedures which affect the integrity of mucosal nerve fibers, namely by asphyxiating the mucosa, by application of a local anesthetic and by removing the mucosa. However, Ginzel (1959) concluded that in the guinea pig ileum the integrity of the mucous membrane was not essential for initiating the peristaltic reflex by gut distension. Our results in the present study agree with those of Ginzel.

In the present study the properties of nerve cells concerning the sensory input in the reflex arc of peristalsis were partly clarified by using the stimulus of gut distension. However, detailed afferent pathways and the identification of nerve cells on them must be studied further.

This study was supported by a research grant from the Ministry of Education, Science and Culture of Japan.

\section{REFERENCES}

Bayliss, W. M. and Starling, E. H. (1899) The movements and innervation of the small intestine. J. Physiol. (Lond.), 24: 99-143.

Bülbring, E., LIN, R. C. Y., and Schofield, G. (1958) An investigation of the peristaltic reflex in relation to anatomical observation. Q. J. Exp. Physiol., 43: 26-37.

Crema, A., Frigo, G. M., and Lecchini, S. (1970) A pharmacological analysis of the peristaltic reflex in the isolated colon of the guinea-pig or cat. Br.J. Pharmacol. Chemother., 39: 334-345.

Frigo, G. M. and LeCCHINI, S. (1970) An improved method for studying the peristaltic reflex in the isolated colon. Br. J. Pharmacol. Chemother., 39: 346-356.

GinzeL, K. H. (1959) Investigations concerning the initiation of the peristaltic reflex in the guinea-pig ileum. J. Physiol. (Lond.), 148: 75P-76P.

Hirst, G. D. S., Holman, Mollie E., and Spence, I. (1974) Two types of neurons in the myenteric plexus of duodenum in the guinea-pig J. Physiol. (Lond.), 236: 303-326.

Vol. 30, No. 2, 1980 
HiRst, G. D. S. and MCKIRDY, H. C. (1974) A nervous mechanism for descending inhibition in guinea-pig small intestine. J. Physiol. (Lond.), 238: 129-143.

Hirst, G. D. S., Holman, Mollie E., and McKirdy, H. C. (1975) Two descending nerve pathways activated by distension of guinea-pig small intestine. J. Physiol. (Lond.), 244: 113-127.

Hukuhara, T., Yamagami, M., and Nakayama, S. (1958) On the intestinal intrinsic reflexes. Jpn. J. Physiol., 8: 9-10.

IGGo, A. (1955) Tension receptors in the stomach and urinary bladder. J. Physiol. (Lond.), 128: $593-607$.

IGGO, A. (1957) Gastro-intestinal tension receptors with unmyelinated afferent fibers in the vagus of the cat. Q.J. Exp. Physiol., 42: 130-143.

NisHi, S. and NoRTH, R. A. (1973) Intracellular recording from the myenteric plexus of the guinea-pig ileum. J. Physiol. (Lond.), 231: 471-491.

Ohrawa, H. and Prosser, C. L. (1972) Electrical activities in myenteric and submucous plexuses of cat intestine. Am. J. Physiol., 222: 1412-1419.

Paintal, A. S. (1954) A study of gastric stretch receptors. Their role in the peripheral mechanism of hunger and thirst. J. Physiol. (Lond.), 126: 255-270.

SchabadAsCH, A. (1930) Intramurale Nervengeflechte des Darmrohres. Z. Zellforsch., 10: $320-385$.

Trendelenburg, P. (1917) Physiologische und pharmakologische Versuche über die Dünndarmperistaltik. Arch. Exp. Pathol. Pharmakol., 81: 55-129.

Woon, J. D. (1970) Electrical activity from a single neuron in Auerbach's plexus. Am. $J$. Physiol., 219: 150-169.

WOOD, J. D. and MAYER, C. J. (1974) Discharge pattern of single enteric neurons of the small intestine of the cat, dog and guinea-pig. In: Proc. IVth Int. Symp. Gastrointestinal Motility, Mitchell Press, Vancouver, Canada, pp. 387-408.

WoodBuRY, J. W. and BRAY, A. J. (1956) Intracellular recording from moving tissues with flexibly mounted electrode. Science, 123: 100-102.

Yokoyama, S., Ozaki, T., and KaJitsuka, T. (1977) Excitation conduction in Aurebach's plexus of the rabbit small intestine. Am. J. Physiol., 232: E100-E108.

Yokoyama, S. and Ozaki, T. (1978) Polarity of effects of stimulation of Auerbach's plexus on longitudinal muscle. Am. J. Physiol., 235: E345-E354. 